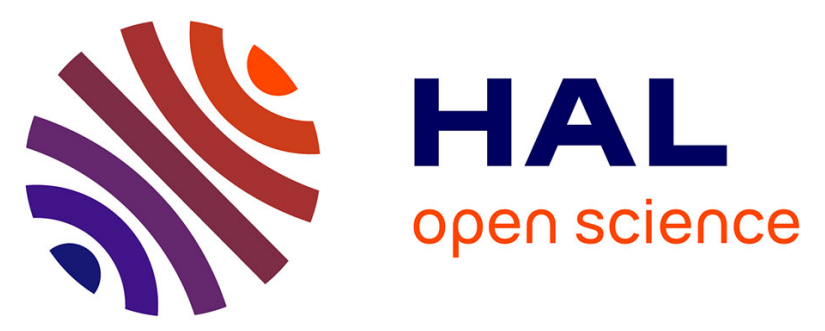

\title{
A Preliminary Study For A Biomechanical Model Of The Respiratory System
}

Jacques Saadé, Anne-Laure Didier, Pierre-Frédéric Villard, Romain Buttin, Jean-Michel Moreau, Michael Beuve, Behzad Shariat

\section{To cite this version:}

Jacques Saadé, Anne-Laure Didier, Pierre-Frédéric Villard, Romain Buttin, Jean-Michel Moreau, et al.. A Preliminary Study For A Biomechanical Model Of The Respiratory System. Engineering and Computational Sciences for Medical Imaging in Oncology - ECSMIO 2010, May 2010, Angers, France. pp.509-515. hal-00509817

\section{HAL Id: hal-00509817 https://hal.science/hal-00509817}

Submitted on 16 Aug 2010

HAL is a multi-disciplinary open access archive for the deposit and dissemination of scientific research documents, whether they are published or not. The documents may come from teaching and research institutions in France or abroad, or from public or private research centers.
L'archive ouverte pluridisciplinaire HAL, est destinée au dépôt et à la diffusion de documents scientifiques de niveau recherche, publiés ou non, émanant des établissements d'enseignement et de recherche français ou étrangers, des laboratoires publics ou privés. 


\title{
A PRELIMINARY STUDY FOR A BIOMECHANICAL MODEL OF THE RESPIRATORY SYSTEM
}

\author{
Jacques Saadé, Anne-Laure Didier, Romain Buttin, Jean-Michel Moreau, Michaël Beuve, Behzad \\ Shariat \\ LIRIS CNRS UMR 5205, Université Claude Bernard Lyon 1, 43 Boulevard du 11 novembre 1918, 69622 Villeurbanne, \\ France \\ jacques.saade, michael.beuve, behzad.shariat@liris.cnrs.fr \\ Pierre-Frédéric Villard \\ LORIA, Nancy University, 615 rue du Jardin Botanique, 54602 Villers les Nancy, France
}

\begin{abstract}
Keywords: Biomechanics, Respiratory system, Finite element method, Motion.
Abstract: Tumour motion is an essential source of error for treatment planning in radiation therapy. This motion is mostly due to patient respiration. To account for tumour motion, we propose a solution that is based on the biomechanical modelling of the respiratory system. To compute deformations and displacements, we use continuous mechanics laws solved with the finite element method. In this paper, we propose a preliminary study of a complete model of the respiratory system including lungs, chest wall and a simple model of the diaphragm. This feasibility study is achieved by using the data of a "virtual patient". Results are in accordance with the anatomic reality, showing the feasibility of a complete model of the respiratory system.
\end{abstract}

\section{Introduction}

Patients internal motions have large implications in different domains such as imaging and treatment (chirurgical operations or radiation therapy). Thereby the respiratory motion reduces the efficiency of radiotherapy benefits. Indeed, thoracic and abdominal tumours can move and deform due to respiration. It is then essential to know their position and shape deformation to be able to optimize the dose to tumour and healthy tissues. Lung tumours are particularly concerned by this motion (Mori et al, 2007; Seppenwoolde et al., 2002). Several management strategies including breath holding (Gagel et al, 2007; Wong et al, 1999), beam gating (Ozhasoglu and Murphy, 2002) and tracking have been discussed in the literature to account for tumour displacement (Giraud et al, 2006). A disadvantage of breath holding and beam gating is that, part of the time, the beam is off. Another disadvantage is that they do not take into account some irregularities in the breathing cycle. Indeed, both methods deduce tumour position from an external breathing parameter (spirometry, abdominal or thoracic height...). Ozhasoglu and Murphy (2002) demonstrated that respiratory compensation strategies that use an external breathing signal to infer tumour position lack the ability to detect and adapt to continuously changing characteristics of respiratory motion during treatment. Other studies (Shirato et al, 2006) show that the respiratory motion has some non-reproducible aspects that need to be taken into account during radiotherapy. This nonreproducibility will be explained later in the anatomy part. Tracking fiducial markers implanted inside the tumour or tracking the tumour using the CyberKnife ${ }^{\circledR}$ robotic radiosurgery system are techniques that take into account the nonreproducibility of the breathing cycle. While the implantation of fiducial markers is an intrusive method that may lead to medical complications such as pneumothorax (Jiang, 2006), the CyberKnife ${ }^{\circledR}$ system has also some inconveniences such like the long treatment time requirement and the irradiation of the patient by the tracking device.

Alternatively we propose a model based solution that takes into account the non-reproducible aspects of breathing motion: a biomechanical modelling of the respiratory system monitored by at least two external parameters (Thoracic motion and spirometry). Ribs and diaphragm displacements can be computed out of thorax outer-surface motion and 
spirometry measurements. Lungs deformations, and then tumour displacements, can be deduced considering the organ interactions. All deformations and displacements are calculated using continuous mechanics laws, solved with the finite element method.

Another requirement, particularly important in the context of hadrontherapy, is the ability to predict not only tumour motion but also motions, deformations and density changes of any tissue traversed by the beam.

Our group has been active in the biomechanical modelling of the respiratory system (Villard et al, 2005, Didier et al, 2007, 2009) and the transformation of biomechanical data into 4D-CT data (Villard et al, 2006). Previously, we have validated a chest wall model based on rib kinematics that enables the computation of rib displacements out of thorax outer-surface motion (Didier et al, 2009). In this paper, we develop a feasibility study of a diaphragm model. First, we summarize previous studies concerning the biomechanical modelling of the respiratory system. Then we expose a complete model of the respiratory system, using a "virtual patient" data, and the preliminary results on the diaphragm and lung motions.

\section{Anatomy of the respiratory system}

Lungs are passive structures that inflate under muscles action. The increase of thoracic volume, due to inspiratory muscles action, induces lung expansion, leading to internal negative pressure and consequently to inspiration. Contact of the lungs with the ribcage and the diaphragm is maintained by the pleura. The pleura is composed of two membranes (figure 1): the first, referred to as parietal, covers the chest wall, the mediastinum and the diaphragm while the second, referred to as visceral, covers the outer surface of the lungs.

The space in between the parietal and visceral pleura, known as the pleural space, is filled with an incompressible fluid which lubricates the pleural space and allows the lungs to easily slide against the chest wall and the diaphragm during their expansion. Lungs mainly expand under the action of the external intercostal muscles (EIM) and the diaphragm. The role of the EIM is relatively important in both quiet and forced respiration. They are inserted between the ribs from the second to the twelfth rib and they are responsible for the rib elevation during inspiration.

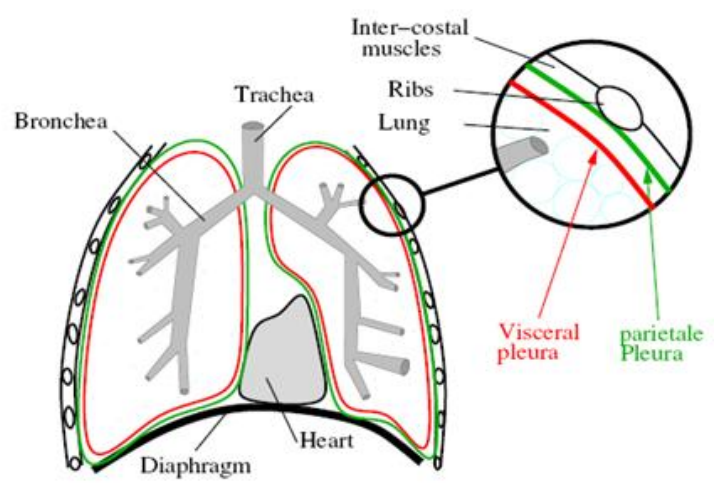

Figure 1: Anatomy of the respiratory system.

The diaphragm is a digastric muscle which separates the thoracic and abdominal cavity. It is composed of two domes (figure 2): the right dome comes up to the fourth intercostal space whereas the left dome remains below the fifth rib. The diaphragm is constituted of a peripheral part (muscular fibre) and a central tendon (figure 2). The peripheral part is linked to the lower thoracic cavity perimeter and has three major insertions: lumbar, sternum and ribs. During inspiration, the muscular contraction fibres bring down the central tendon. This lowering increases the vertical diameter of the thorax. As for the lungs, EIM action induces postoanterior and transversal inflation while the diaphragm action causes vertical motion. The EIM and the diaphragm may act independently, making respiration a non-reproducible and an unpredictable movement. Thereby, in general, lung motion cannot be simply predicted by a correlation with one single parameter.

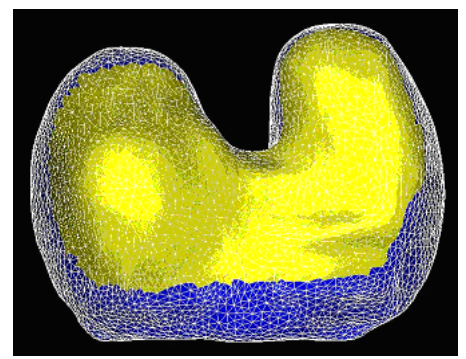

Figure 2: A mesh representation of the diaphragm (upper view). The central tendon is represented in yellow whereas the peripheral part is in blue. 


\section{Biomechanical modelling of the respiratory system}

In the past, several studies were achieved to model the lung environment. A description of these studies can be found in Baudet et al. (2003), Villard et al. (2005) and Al-Mayah et al. (2007). Thus, we focus on the more recent studies. Villard et al. (2005) used a patient's CT scan images to build the geometry of a finite element lung model. They also studied lung motion sensitivity to Poisson's ratio, elastic modulus (Young modulus) and contact conditions at the pleura. It appeared that Poisson's ratio has an effect on the amplitude and the orientation of the displacements while Young modulus should be carefully chosen because a change in its value may either induce a faulty implementation of contact conditions or increase the computing time needs. Brock et al. (2005) developed a platform to perform multi-organ deformable image registration using finite element modelling. The model was developed using images from magnetic resonance (MR) scanning. The lungs were included in this model and good agreement was found between the finite element simulation using orthogonal displacement (OD) and the MR data. Didier et al. (2007) showed the significant role of the pleura and the necessity to include its effect in the model. The role of the pleura is simulated by applying contact conditions that allow sliding on the lungs surface (contact without friction). The results of this model were compared to those of the OD model and showed an improvement in predicting the position of the lungs while the computing time requirement was higher. Al-Mayah et al. (2007) included the role of the pleura and two other nonlinearities to the model (hyperelastic nonlinear materials and nonlinear geometry due to large displacements). They also added the chest wall and the tumour to the model and achieved a good precision on lung and tumour position. An inconvenience of adding the nonlinearities was the increase in the computation time needed to complete the simulation. All the models mentioned above, focused on deformable registration of soft tissues in the thorax and did not invest in the bone tissues of the rib cage. Going from the fact that the bones of the rib cage can relatively be considered as rigid bodies, Didier et al. (2007) introduced a rigid transformation that simulates the kinematic behaviour of the rib cage intead of simulating the action of each intercostal muscle. The transformation is based on the Finite Helical Axis (FHA) method. Didier et al (2009) developped this method in order to build a model of the chest wall. This model proposed a correlation between ribs motion and thorax-outer surface motion and achieved a good precision on lungs position in the upper thorax. This model is essential to enable monitoring of lung motion out of thorax motion. Thus, to be able to build a complete model of the respiratory system and to include a second parameter to the monitoring process of the lungs, we introduce in this paper a biomechanical study of the diaphragm and some preliminary results concerning a finite element simulation of its motion.

\section{Diaphragm biomechanical model}

In order to build a complete model of the respiratory system, the diaphragm is modeled and added to the thorax and the lung models. As mentioned in the anatomy part, the diaphragm is composed of a peripheral part (muscular fibre) and a central tendon. During inspiration, muscular fibres contract under the action of a force $F$ and the ribs undergo a displacement $D c$. Both actions cause the lowering of the central tendon (figure 3).

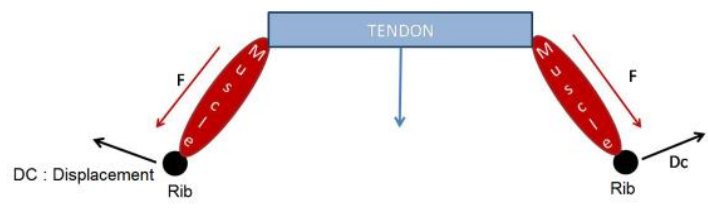

Figure 3: An illustration of the diaphragm motion.

Since most thoracic medical imaging data cover only the lungs and big parts of the diaphragm are not included in the imaging protocol, we chose to carry out our study on a "virtual model" data. We chose the data of a virtual patient that include surface meshes of the skin, the ribcage, the lungs and the diaphragm. Figure 4 shows the different mesh data.

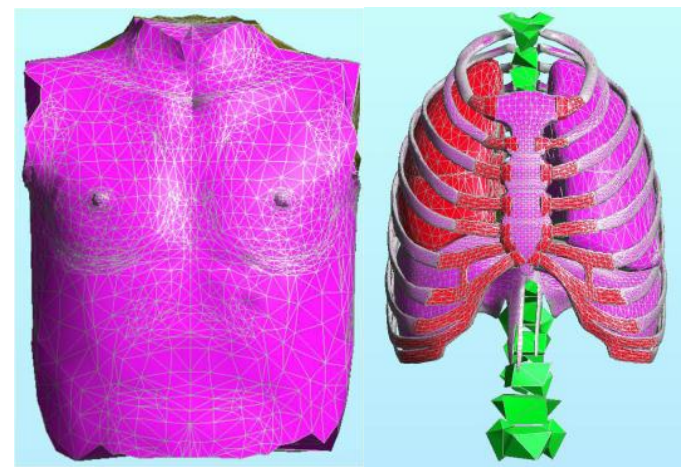

Figure 4: Anatomic elements of the model. 
We apply on this geometrical model the mechanical laws and boundary conditions and material properties as follow.

\subsection{Mechanical behaviour laws}

We use continuous mechanics laws to compute the deformations with a non-linear behaviour law that allows large displacements. We considered elastic materials. Calculations were made in the static mode which means that only the initial and final states of deformations are computed after the application of boundary conditions.

\subsection{Boundary conditions}

The boundary conditions of our model are illustrated in figure 5. They are the same as described in Didier et al (2009), but we add the boundary conditions at the diaphragm. Lungs are fixed near the trachea and the pleura behaviour is simulated by applying contact conditions allowing lungs surface to slide against the chest wall (parietal pleura). These contact conditions permit us to model the negative intra-pleural pressure, and the sliding surface represents the pleural fluid. According to reality, parietal pleura is directly linked to the ribs or fat tissue. A particular rigid transformation $(D c)$ computed with the finite helical axis method is applied to each rib. Ribs are directly linked to the fat tissues which are also directly linked to the skin. The diaphragm boundary conditions are as follow:

\subsubsection{Diaphragm / Ribcage}

As mentioned in the anatomy part, the diaphragm is attached to the ribcage. In our simulation, we made sure, in the attached region, that the corresponding nodes were linked together.

\subsubsection{Diaphragm / Fat tissues, Diaphragm / Parietal pleura}

The diaphragm is directly linked to the fat tissues and to the parietal pleura. This is an approximation because normally the fat tissues can slide against the diaphragm. The soft tissues that lie under the diaphragm represent a resistance to the force that tends to lower the diaphragm. They are simply modelled like the fat tissues and they are affected the same biomechanical parameters.

\subsubsection{Contraction of the muscular fibres}

To mimic the contraction, we apply the force $\mathrm{F}$ illustrated on figure 5. This force should be parallel to muscle orientation. In a first approximation, we apply vertical (cranio-caudal) forces that are oriented downwards. These forces are applied on each node of the muscular part of the diaphragm. This choice was motivated by the goal of this work, which aims at demonstrating that it is possible to propose a complete model of the respiratory system as soon as a diaphragm model is available.

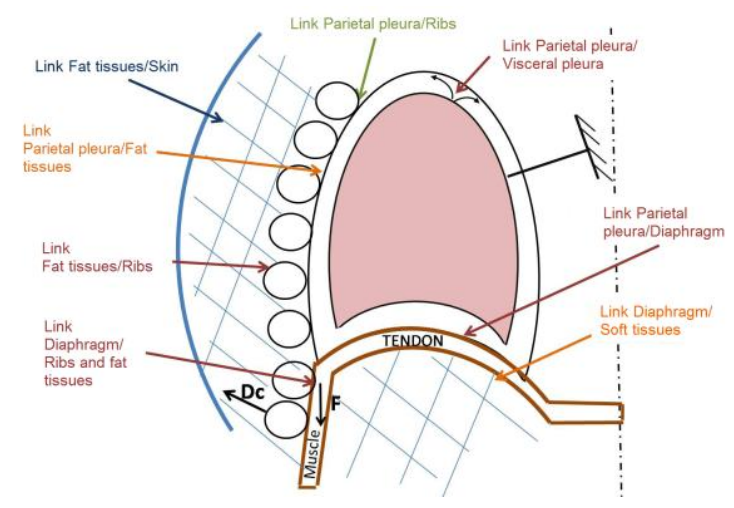

Figure 5: Boundary conditions.

\subsection{Material properties}

Table 1 illustrates the biomechanical parameters (Young modulus and Poisson's ratio) of the lungs, ribs, fat tissues, skin and diaphragm. The diaphragm has two different parts (muscle and tendon) and each part has different mechanical properties. All parameters are taken from the bibliography (Handriks, 2001; Promayon and Baconnier, 2008).

Table 1: The biomechanical properties of the different

\begin{tabular}{ccc} 
& organs. & \\
\hline & Lungs & $\begin{array}{c}\text { Ribs \& } \\
\text { vertebrae }\end{array}$ \\
\hline $\begin{array}{c}\text { Young } \\
\text { Modulus } \\
(\mathrm{MPa})\end{array}$ & $700 * 10^{-6}$ & 5000 \\
\hline $\begin{array}{c}\text { Poisson's } \\
\text { ratio }\end{array}$ & 0.3 & 0.3 \\
\hline $\begin{array}{c}\text { Young } \\
\text { Modulus } \\
\text { (MPa) }\end{array}$ & Fat tissues & Skin \\
\hline $\begin{array}{c}\text { Poisson's } \\
\text { ratio }\end{array}$ & 3 & 3 \\
\hline
\end{tabular}




\begin{tabular}{ccc}
\hline & $\begin{array}{c}\text { Diaphragm } \\
\text { (Muscle) }\end{array}$ & $\begin{array}{c}\text { Diaphragm } \\
\text { (Tendon) }\end{array}$ \\
\hline $\begin{array}{c}\text { Young } \\
\text { Modulus }\end{array}$ & 5.32 & 10 \\
$\begin{array}{c}\text { PPa) } \\
\text { Poisson's ratio }\end{array}$ & 0.3 & 0.3 \\
\hline
\end{tabular}

\section{Results}

The "virtual patient" data are available for only one respiratory state (we consider it arbitrarily as the exhalation state). Because we need at least two respiratory states to be able to compute rib kinematics parameters (Didier et al, 2009), we apply the rib parameters computed for a real patient in a previous study (Didier et al, 2009). We apply the force $F$ as explained in 4.2 .3 , then we compare the diaphragm at the initial state with the diaphragm at the computed state to deduce its deformation and the displacement fields. All calculations are made using Code-Aster finite element software (http://www.code-aster.org). The results (deformations and displacement fields) are visualised using GMSH software (http://www.geuz.org/gmsh) and MESH (Aspert et al, 2002).

Figure 6 shows the diaphragm deformations between the initial state (wireframe mesh) and the computed final state (colored mesh). We can observe that the central tendon goes down due to the action of the force applied to the muscular part. We observe also a lateral augmentation of the diaphragm diameter due to the rib motion.

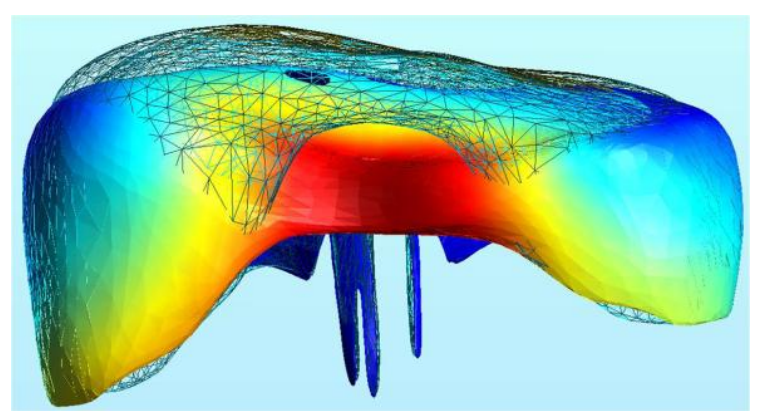

Figure 6: Frontal view of the diaphragm motion between exhalation (wireframe mesh) and computed inhalation state (coloured mesh). The deformations are illustrated in colours on the computed mesh with red representing the highest deformation value and blue its lowest value. The colour scale is proportional to the motion amplitude.
Figure 7 represents the displacement vectors at different locations on the diaphragm. The two red vectors represent the mean orientation of the displacement vectors at the level of the central tendon (upper view) and at the level of the lower muscular part (lower view). On the muscular part, the displacement is lateral, oriented downwards while the displacement on the level of the central tendon tends to be more vertical. Globally, these results are in accordance with the anatomy. Indeed, the central tendon is relatively rigid, and therefore less influenced by the ribs motion. However, after the contraction of the muscular part, the central tendon goes down while preserving the form of its domes. This result is in accordance with the bibliography and the observations made by Boriek and Rodarte (1997).

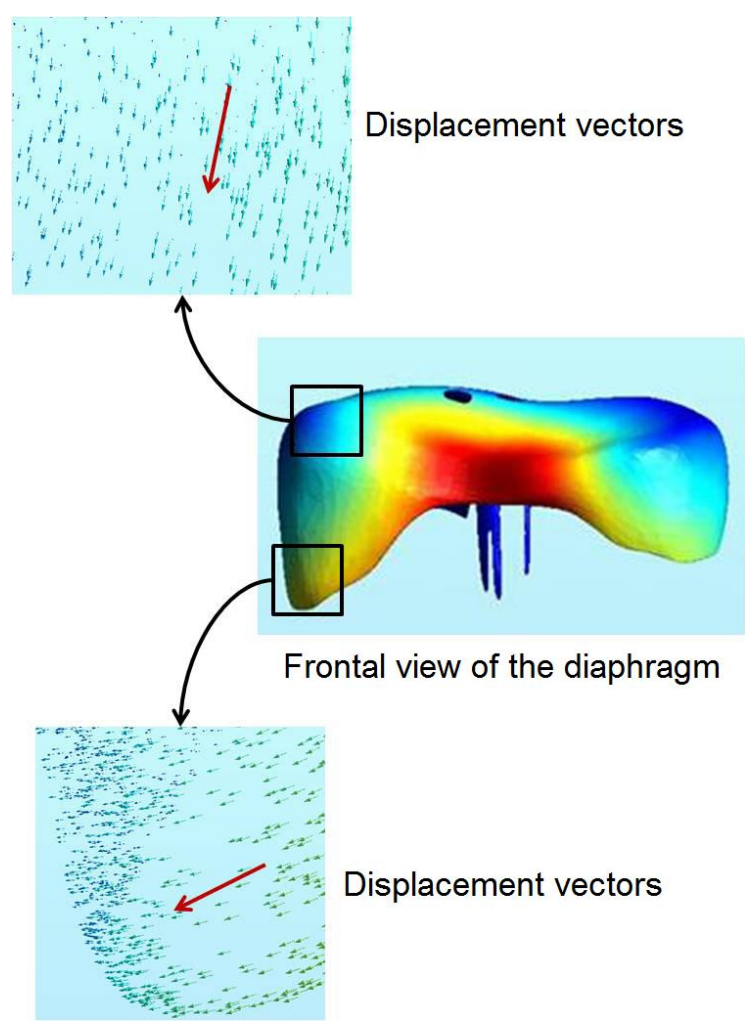

Figure 7: Motion of the diaphragm.

We also present the results of our model concerning lungs motion. Figure 8 represents the displacement vectors at different locations on the lungs. The two red vectors represent the mean orientation of the displacement vectors at a high level of the lungs (upper view) and at a low level of the lung near the diaphragm (lower view). On the upper level, the displacement is lateral, oriented upwards while the displacement on the lower level 
tends to be more vertical and is oriented downwards. These results are in accordance with the anatomy. Indeed, the higher parts of the lungs are influenced by the ribs motion (lateral motion with elevation) and the lower parts are more influenced by the motion of the diaphragm central part (downwards).

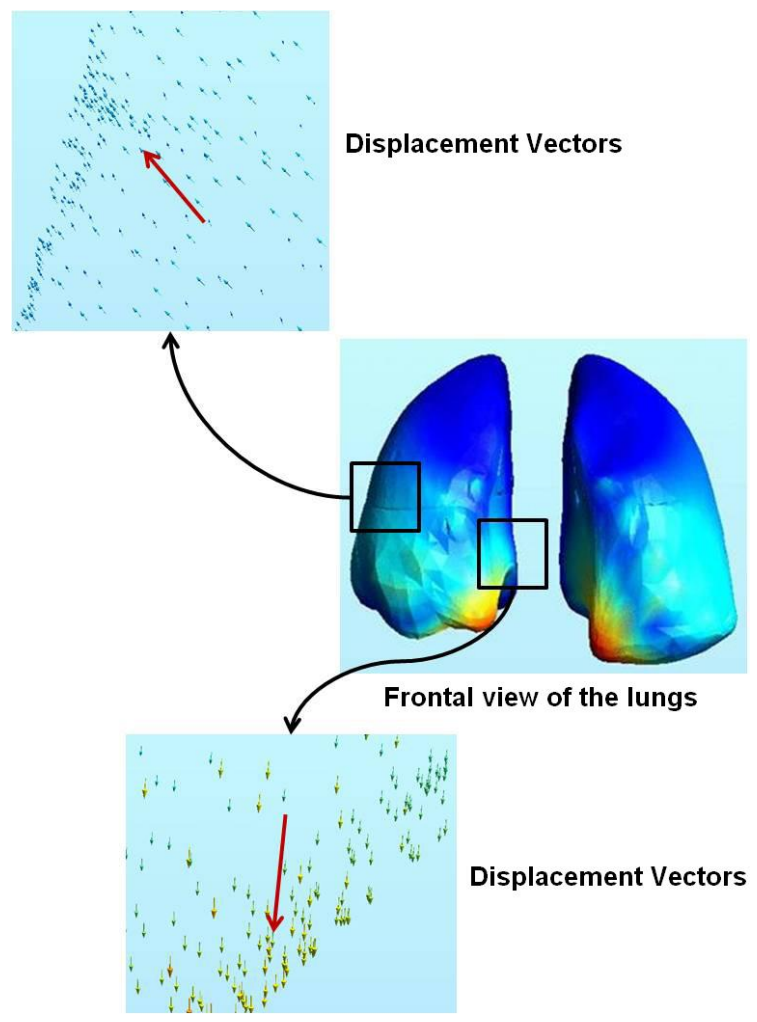

Figure 8: Lungs motion.

\section{Conclusions}

We have developed a complete model of the respiratory system built from "virtual patient" data. A simple model of the diaphragm, which was missing in our previous studies, has been introduced. We showed preliminary results, which are in accordance with the anatomical reality. The central part of the diaphragm tends to move downwards, while the muscular part motion tends to be lateral oriented downwards due to rib motions. In future works, the model should be applied on a real patient's data to enable quantitative comparisons of the results. The applied nodal forces should be replaced by more appropriated formulations. In particular, the forces could be parallel to muscular fibres in agreement with anatomy and derived from biomechanical considerations. Last, the forces should be correlated to thoracic motion and air flow to the lungs.

\section{Acknowledgments}

We thank the French League against Cancer and PRRH ETOILE for their financial support.

\section{References}

A. Al-Mayah, J. Moseley, and KK. Brock. Contact surface and material nonlinearity modeling of human lungs. Physics in medicine and biology, 53 : 305-317, 2008.

N. Aspert, D. Santa-Cruz, and T. Ebrahimi. Mesh : measuring errors between surfaces using the hausdorff distance. IEEE conference in multimedia and expo (ICME), pages 705-708, 2002.

A.M. Boriek and J.R. Rodarte. Effects of transverse stiffness and central tendon on displacement and shape of a simple diaphragm model. Journal of applied physiology, 82(5) : 1626-1636, 1997.

KK. Brock, MB. Sharpe, LA. Dawson, SM. Kim, and DA. Jaffray. Accuracy of a finite element model-based multiorgan deformable image registration. Medical Physics, 32(6) : 1647-1659, 2005.

A.L. Didier, P.F. Villard, J.Y. Bayle, M. Beuve, and B. Shariat. Breathing thorax simulation based on pleura behaviour and rib kinematics. Information Visualisation, IEEE Computer Society, pages 35-40, 2007.

A.L. Didier, P.F. Villard, J. Saadé, J.M. Moreau, M Beuve, and B. Shariat. A chest wall model based on rib kinematics. Information Visualisation, IEEE Computer Society, pages 159-164, 2009.

B. Gagel, C. Demirel, A. Kientopf, M. Pinkawa, M. Piroth, S. Stanzel, C. Breuer, B. Asadpour, T. Jansen, R. Holy, J.E. Wildberger, and M.J. Eble. Active breathing control $(\mathrm{abc})$ : determination and reduction of breathinginduced organ motion in the chest. Int. J. Radiation Oncology Biol. Phys., 67(3) : 742-749, 2007.

P. Giraud, E. Yorke, S. Jiang, L. Simon, K. Rosenzweig, and G. Mageras. Reduction of organ motion effects in IMRT and conformal $3 \mathrm{~d}$ radiation delivery by using gating and tracking techniques. Cancer radiothérapie, $10: 269$ $282,2006$.

F. M. Hendriks. Mechanical behaviour of human skin in vivo - a literature review. In Nat.Lab. Unclassified Report 820. Philips Research Laboratories, 2001. 
S.B. Jiang. Radiotherapy of mobile tumors. Seminars in Radiology Oncology, 16 : 239-248, 2006.

S. Mori, M. Endo, S. Komatsu, T. Yashiro, S. Kandatsu, and M. Baba. Four-dimensional measurement of lung tumor displacement using 256-multi-slice ctscanner. Lung Cancer, 56 : 59-67, 2007.

C. Ozhasoglu and M.J. Murphy. Issues in respiratory motion compensation during external-beam radiotherapy. Int. J. Radiation Oncology Biol. Phys., 52(5) : 1389-1399, 2002.

E. Promayon and P. Baconnier. A 3D discrete model of the diaphragm and human trunk. ESAIM Proceedings, 23 : 66-77, 2008.

Y. Seppenwoolde, H. Shirato, K. Kitamura, S. Shimizu, M. Van Herk, J.V. Lebesque, and K. Miyasaka. Precise and real-time measurement of $3 \mathrm{~d}$ tumor motion in lung due to breathing and heartbeat, measured during radiotherapy. Int. J. Radiation Oncology Biol. Phys., 53(4) : 822-834, 2002.

H. Shirato, K. Suzuki, GC. Sharp, K. Fujita, R. Onimaru, M. Fujino, N. Kato, Y. Osaka, R. Kinoshita, H. Taguchi, S. Onodera, K. Miyazaka. Speed and amplitude of lung tumor motion precisely detected in four-dimensional setup and in real-time tumor-tracking radiotherapy. Int. J. Radiation Oncology Biol. Phys., 64(4) : 1229-1236, 2006.

P.F. Villard, M. Beuve, and B. Shariat, V. Baudet, and F. Jaillet. Simulation of lung behavior with finite elements : influence of bio-mechanical parameters. Information Visualisation, IEEE Computer Society, pages 65-70, 2005.

P.F. Villard, M. Beuve, and B. Shariat : An Approach to Convert 4D Geometry into a 4D CT Scan. WSCG (Winter School of Computer Graphics), UNION Agency ed. Plzen (Czech Republic). pp. 163-170. ISBN 80-86943-05. 2006.

J.W. Wong, M.B. Sharpe, D.A. Ja_ray, V.R. Kini, J.M. Robertson, J.S. Stromberg, and A.A. Martinez. The use of active breathing control (ABC) to reduce margin for breathing motion. Int. J. Radiation Oncology Biol. Phys., 44(4) : 911-919, 1999. 